\title{
Sexualidade, saúde e contextos: influência da cultura e etnia no comportamento sexual
}

Fernanda Fonseca, ${ }^{*}$ Marisa Cortez Lucas**

\section{RESUMO}

O comportamento sexual é uma realidade complexa. Não é apenas biologicamente que somos sexuados, pois toda a nossa organização social e cultural é, também, sexuada. É pretensão deste artigo abordar algumas das implicações étnicas e culturais sobre os comportamentos sexuais da nossa população, a fim de avaliar o reflexo que podem ter na prática dos prestadores de cuidados de saúde

Nos vários momentos da vida do indivíduo, encontram-se algumas particularidades que permitem confirmar a importância da cultura e da etnia no comportamento sexual.

Embora a vertente socioeconómica se constitua como um factor importante na sexualidade adolescente, a etnia e a cultura podem introduzir algumas especificidades. Na adolescência pode verificar-se que os sentimentos relativamente ao coito, número de parceiros sexuais, uso do preservativo e gravidez indesejada, podem depender de elementos associados à ascendência, à etnia e à cultura.

Também na idade adulta, esses elementos vão influenciar os comportamentos sexuais. Quando se abordam, entre outros, a imagem face ao corpo, a menstruação, a sexualidade marital, a masturbação, a gravidez, as infecções sexualmente transmissíveis e o uso do preservativo, verifica-se que a etnia e a cultura vão condicionar diferenças entre os vários grupos.

Por outro lado, a sexualidade é sempre regulada, em algum grau, pelas sociedades e está sujeita às mudanças históricas, demográficas, culturais e económicas. O género vai influenciar, de forma decisiva, os comportamentos de todas as etnias e culturas. Por exemplo, a saúde sexual e reprodutiva e a contracepção continuam a ser uma responsabilidade atribuída às mulheres.

De facto, em todo o mundo, uma grande percentagem de homens casados refere nunca ter discutido planeamento familiar com as suas parceiras e menos de um terço é responsável pela contracepção.

Podemos concluir que é importante que os técnicos de saúde estejam esclarecidos sobre como o sistema de crenças e atitudes característico das diferentes comunidades influencia os comportamentos sexuais.

Palavras-Chave: Sexualidade; Comportamento Sexual; Atitude Sexual; Saúde Sexual; Etnia.

\section{INTRODUÇÃO}

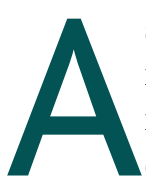

sexualidade é uma realidade complexa. O nosso corpo é sexuado e somos biologicamente sexuados. A sexualidade está enraizada no biológico e os nossos comportamentos sexuais dependem dessa dimensão. Por outro lado, a própria actividade sexual produz alterações fisiológicas. No entanto, não somos só biologicamente sexuados,

*Assistente Graduada de MGF; Pós-graduação em Sexualidade Humana **Professora do Ensino Secundário, Formadora; Pós-graduação em Sexualidade Humana toda a nossa organização social e cultural são também sexuados. ${ }^{1}$ Esta tríade irá influenciar, como um todo, a sexualidade, nas suas mais variadas vertentes. Em Portugal, um país que integra, actualmente, populações de várias culturas, ainda não existem estudos suficientes que permitam enquadrar, de forma clara e concisa, a sexualidade nas vertentes étnica e cultural.

Neste artigo, pretende-se abordar algumas das implicações das diferenças culturais sobre os comportamentos sexuais da nossa população e o reflexo que podem ter na nossa prática como prestadores de cuida- 
dos de saúde. Em suma, perspectiva-se a sexualidade na complexidade de vários vectores que influenciam, de forma decisiva, a nossa forma de compreensão e intervenção na prática diária.

\section{ADOLESCÊNCIA - UM MOMENTO MULTIDIMENSIONAL}

Sendo a sexualidade uma dimensão vivencial, onde interagem os contextos biológico, psicológico, social e cultural, torna-se importante perceber até que ponto os comportamentos sexuais estão codificados por variáveis construídas em universos multidimensionais. Esta multidimensionalidade torna-se particularmente importante no caso da sexualidade dos adolescentes. De facto, é neste período que se vão estruturar as bases comportamentais que modelarão os comportamentos sexuais futuros. ${ }^{2}$ Assim, é necessário entender até que ponto os vectores etnia, cultura e sociedade são essenciais à compreensão da natureza multidimensional da sexualidade.

Neste contexto, afigura-se uma dificuldade: etnia e cultura inserem-se em contextos conexos e interdependentes, mas não significam exactamente o mesmo. De facto, uma etnia tem as suas características específicas, mas a sua inserção numa determinada cultura, ou seja, em valores, hábitos e tradições que formam um determinado sistema social, pode modelar traços supostamente associados e definidos numa etnia. ${ }^{1-3}$

É aqui que, na prática clínica, se exige um aprofundamento do conhecimento das características que fazem parte das dimensões étnica e cultural de cada indivíduo. Devem, portanto, analisar-se, os contextos específicos de cada etnia, sem, no entanto, esquecer, que cada indivíduo está inserido numa cultura, que também lhe condiciona o comportamento. Naturalmente que esta influência se estende à sexualidade.

Na adolescência, a acentuada influência nos comportamentos sexuais depende muito das condições socioeconómicas e da aculturação. De facto, os adolescentes, ainda que identificados com a sua etnia, interiorizam os hábitos do meio social e cultural que os envolve, através de um processo de socialização. Um dos exemplos paradigmáticos é o duplo padrão de género, ${ }^{4}$ que corresponde à diferente atitude da sociedade, face aos comportamentos sexuais, em função do género. Pode-se, portanto, afirmar que o duplo padrão carac- teriza e molda, de forma decisiva, a sexualidade, influenciando os traços referentes à etnia.

Interessa, ainda, abordar os comportamentos sexuais de risco na adolescência, cujos índices preocupantes determinam a ocorrência das infecções sexualmente transmissíveis e da gravidez não desejada, entre outras. ${ }^{5,6}$

Toda a informação direccionada à adolescência sobre os riscos do VIH, a afectividade na sexualidade, a gravidez adolescente, entre outros, demonstra que o sistema de valores reflecte, em muito, aquilo que uma determinada Cultura e Sociedade definem como a prática socialmente aceite. Neste contexto, surge um vector muito importante e talvez o mais complexo de toda a vivência adolescente: a identidade sexual, que, necessariamente, caracterizará o seu comportamento sexual, com toda a diversidade que implica. Perspectivar esta construção obriga a estar sensível a todos os elementos que fazem parte da vida do adolescente. Aqui, o clínico terá que considerar como base da sua abordagem ao adolescente as vivências do meio social, cultural e étnico, ou seja, as experiências com o mundo dos adultos e dos pares. Neste âmbito, deve dar-se particular atenção às expectativas.

A actuação do adolescente passa por aquilo que é esperado face ao seu «desempenho»/comportamento sexual. Quando existe a referência a estas expectativas, que podem actuar como elementos prescritores da sexualidade, estão-se a pressupor dois vectores: o papel sexual (as diferenças associadas ao género) e a forma como esse papel é codificado pelo grupo de pares, no conjunto de características da sexualidade. Por exemplo: um adolescente cuja vivência decorre num grupo onde a sexualidade, ou qualquer aspecto particular desta (p. ex. sentimentos negativos ou positivos face ao coito), é valorizada, irá, certamente, reflectir essa característica. Neste aspecto, surgem alguns dados interessantes. Pode-se verificar que os rapazes afro-americanos, em comparação com os brancos, vivenciam a sexualidade com menos sentimentos de vergonha ou culpa. Porém, de uma forma bastante curiosa, não se verificaram diferenças entre as raparigas brancas e afro-americanas. ${ }^{7}$

Também existem variáveis associadas à etnia e ao género que influenciam o início da actividade sexual. Num estudo realizado nos Estados Unidos, é bem pa- 
tente a diferença de comportamentos: aos 17 anos, verificou-se que $40 \%$ das raparigas afro-americanas já eram sexualmente activas, enquanto que o mesmo se verificava, apenas, em $25 \%$ das euro-americanas e $24 \%$ das de origem latina. ${ }^{8}$ Assim, pode concluir-se que as afro-americanas representavam um grupo com um início mais precoce da actividade sexual, por comparação com os outros grupos étnicos estudados.

Os adolescentes do sexo masculino expressam uma maior tendência a ter múltiplos parceiros. Os adolescentes negros têm, também, maior probabilidade de ter múltiplos parceiros, por comparação com as outras etnias. Existem, ainda, diferenças no uso do preservativo: os adolescentes negros e brancos apresentam uma maior probabilidade de uso do preservativo, quando comparados com os hispânicos. Note-se, porém, que estes resultados são também influenciados pelas condições socioeconómicas. Assim, idade, género, etnia e condições socioeconómicas, constituem-se como factores que, correlacionados entre si, vão influenciar os resultados, no que concerne aos comportamentos sexuais de risco na adolescência. ${ }^{9}$

Sendo Portugal um país multicultural e, portanto, marcado por comunidades migrantes, torna-se importante analisar as dimensões psicossociais e culturais da infecção por VIH/SIDA, onde interagem o comportamento individual e as realidades económica e social, ou seja, factores que envolvem a vida do adolescente e que podem influenciar as suas crenças, atitudes e comportamentos face ao VIH/SIDA.

Num estudo realizado com adolescentes em Portugal, numa comunidade migrante essencialmente oriunda dos PALOP, existem dados que remetem para o facto desta comunidade afirmar, em maior percentagem, já ter tido relações sexuais, enquanto que os portugueses são aqueles que mais referiram ter utilizado o preservativo. Porém, não se verificaram diferenças significativas entre portugueses e a comunidade migrante, face ao conhecimento dos modos de transmissão do VIH. ${ }^{10}$

Na sequência, surgem-nos outros estudos que indiciam diferenças significativas quando se compara caucasianos e negros. Os negros revelam um período mais curto de comportamentos não coitais, quando comparados com os caucasianos. De facto, quando se compara o tempo que dista entre os comportamentos não coitais e os coitais, as raparigas negras apresentaram um valor de $41,4 \%$, quanto a uma mais rápida transição, face aos $28 \%$ verificados nas caucasianas. ${ }^{11}$ Assim, pode concluir-se que as raparigas negras transitam mais rapidamente para comportamentos coitais, quando comparadas com as caucasianas. Daqui decorre a probabilidade das negras iniciarem mais cedo os comportamentos coitais, aumentando, assim, os riscos associados à sexualidade na adolescência.

Associada a esta problemática, surge, inevitavelmente, a gravidez indesejada. De facto, as raparigas afro-americanas apresentam uma maior probabilidade de engravidar sem o desejar, comparativamente a outras etnias. $^{7}$

Nestes estudos, não só se evidencia a necessidade de abordar esta problemática abrangendo as variáveis associadas à multiculturalidade, como também são identificados factores de risco como a pobreza, a exclusão social, as competências sociais e pessoais, entre outras. Basta pensar nas condições socioeconómicas da maioria da comunidade migrante em Portugal, para se enfatizar a necessidade de uma abordagem muito específica a este contexto.

\section{ATITUDES E COMPORTAMENTOS FACE À SEXUALIDADE E SUA IMPORTÂNCIA EM SAÚDE SEXUAL NO ADULTO}

No adulto, os estudos sobre a influência da variável etnia nas questões da saúde e da sexualidade são escassos e deparam-se com dificuldades em estabelecer a causalidade, devido à globalização e miscigenação resultante dos fluxos migratórios. Segundo Danille Gradin, da United Nations Population Fund (UNFPA), «A migração dos nossos dias apresenta uma grande complexidade, sendo um desafio não só para quem migra e como migra, mas, também, no âmbito da Saúde Pública. Este aspecto é inerente às próprias movimentações das pessoas e ao contacto de cada sujeito com determinados factores de saúde (individuais e ambientais), que ficam ligados de um país para o outro».. ${ }^{12}$

Actualmente, com o aumento do número de mulheres migrantes nas últimas décadas, assistimos a um fenómeno recente, que é a feminização das migrações internacionais. Segundo a OMS, dos 191 milhões de migrantes, 95 são mulheres. Em Portugal, a reunificação familiar foi invocada em $23,9 \%$ dos estrangeiros que 
pediram autorização de residência entre 1999 e 2001. Para estas mulheres, a migração pode ser um factor positivo de mudança nas relações de género, com melhoria na igualdade e direitos da mulher que migra, fundamentalmente quanto ao acesso aos serviços de saúde, educação e acção social. Os serviços dos países de acolhimento não se devem esquecer de ter em conta o estatuto ou papel da mulher nas comunidades de origem. ${ }^{12}$

A diversidade de comunidades de origem implica a existência de contextos diversos, não só genéticos, mas também culturais, educacionais e sociais, confrontando-nos com o facto de, perante indivíduos de outras comunidades, existirem diferenças no comportamento sexual, que terão que ser tidas em conta na prática clínica.

\section{ATITUDES FACE AO CORPO}

Regista-se, em todas as culturas, um duplo padrão sexual que, tradicionalmente, restringe a excitação e o comportamento sexual da mulher, enquanto encoraja o do homem. Decorrente deste duplo padrão sexual, as proibições sociais contra a nudez em público pretendem reduzir os estímulos desencadeantes de excitação sexual em público, considerada perigosa para a moral e ordem social, que recai, sobretudo, sobre as mulheres. Encontrava-se presente na nossa cultura e ainda persiste nas comunidades de países islâmicos. Nestas, impõe-se que o corpo da mulher esteja totalmente coberto em público. Decorrente deste facto, as mulheres apresentam-se nas consultas sempre acompanhadas pelo marido ou familiar chegado e nas consultas de planeamento familiar só se deixam observar por mulheres. $^{13}$

\section{MENSTRUAÇÃO}

Em muitas culturas e em vários períodos históricos, a menstruação foi vista com receio, como algo desagradável e passível de controlo moral. Segundo as Escrituras Judaico-Cristãs, a menstruação da mulher é impura, assim como é quem tocar na sua cama (Levítico 15: 19-21). Na tradição Ortodoxa Judaica, a mulher deve dormir separada do marido vários dias, durante e após a menstruação, até se submeter a um banho de limpeza (mikvah). ${ }^{2}$

O teólogo cristão Santo Agostinho pensava que as relações sexuais com uma mulher menstruada eram pe- cado. O Corão (versículo 2: 222) proíbe o sexo com mulher menstruada, assim como, segundo certas interpretações, pode ser impedida de rezar, cozinhar ou entrar na mesquita.

A noção de que a mulher deve evitar ter relações durante a menstruação ainda está muito difundida, mesmo na cultura ocidental dos nossos dias. Cerca de setenta a oitenta por cento dos homens e mulheres evitam-nas durante esse período (Barnhait e col., 1995 e Tanfer e Aral, 1996). Estes autores encontraram vinte por cento de mulheres nos EUA que referem ter actividade sexual durante o período menstrual; tratavam-se, sobretudo, de mulheres brancas de nível educacional mais alto. No entanto, a maioria envolve-se em formas não coitais de relação sexual. ${ }^{2}$

\section{MASTURBAÇÃO}

A frequência com que as pessoas se masturbam varia muito, consoante o sexo, a idade e a etnia. Assim, masturbam-se mais os homens que as mulheres, os mais novos mais que os idosos e, em função da etnia, os afro-americanos menos que os hispânicos, os brancos ou os asiáticos. A influência da idade e do sexo é importante, mas os factores culturais influenciam bastante, sendo a sua prática pouco usual nas sociedades não ocidentais. Algumas culturas têm tabus contra a masturbação masculina, devido às crenças de que poderia causar doença psiquiátrica, como se pensava na nossa cultura até princípios do século XX, ou causar perda de energia yang, segundo a cultura oriental. ${ }^{2}$

\section{FREQUÊNCIA DE ACTIVIDADE SEXUAL}

Os muçulmanos e hindus referem relações sexuais menos frequentes que os ocidentais. Nalgumas culturas, 0 número total de dias de restrição à actividade sexual, de causa religiosa, chega a ser de cem dias por ano. Os muçulmanos e, sobretudo, os hindus, acreditam que o sémen é uma fonte de energia que não deve ser desperdiçada. ${ }^{2,13}$

\section{SEXUALIDADE PRÉ-MARITAL}

Os países muçulmanos mantêm, ainda, fortes restrições à sexualidade pré-marital. ${ }^{13}$

Todas estas normas surgiram num contexto histórico em que a fecundidade era um valor máximo em todos os grupos humanos. Como não existiam métodos 
eficazes de evitar a gravidez não desejada, as relações sexuais pré-matrimoniais eram relações de alto risco. Dado que aqueles que não tinham um compromisso estável não podiam oferecer garantia de cuidados adequados à descendência, considerava-se que não deviam ter relações sexuais. Esta situação mudou, nos finais do séc. $\mathrm{XX}$, devido a quatro factores: a diminuição da mortalidade infantil e o aumento da esperança média de vida, o desenvolvimento dos métodos contraceptivos, a visão mais positiva da sexualidade e a maior permissividade no Ocidente. De facto, a diminuição da mortalidade infantil e o aumento da esperança média de vida levaram ao aumento do número de países a aceitarem o controlo da natalidade. O desenvolvimento dos métodos contraceptivos reduziu o risco de gravidez não desejada. Por outro lado, neste último século, a sexualidade passou a ser vista como uma dimensão positiva e a regulação social dos comportamentos sexuais passou, no Ocidente, a ser mais permissiva. Finalmente, com o advento das democracias, também as relações que tenham lugar entre adultos livres e que não provoquem dano social evidente passam a ser aceites. Na nossa sociedade, mantém-se, no entanto, a regulação dos comportamentos sexuais nos seguintes aspectos: dentro do matrimónio, se os membros do casal entram em conflito; na família, com a proibição do incesto; no controlo de escândalos públicos, nos abusos sexuais a menores e na violência sexual.

\section{ESTRUTURA FAMILIAR}

Em estudos efectuados nos E.U.A., as famílias monoparentais são mais frequentes entre os negros, correspondendo, sobretudo, a mães sozinhas com filhos. ${ }^{2}$ Este aspecto poderá resultar do facto de, em alguns países africanos, a multiplicidade de parceiras nos homens ser aceite e considerada uma prática comum, característica do comportamento sexual masculino. ${ }^{14}$ Face a uma gravidez não desejada ou à mudança de companheira, as mães vão ficando sozinhas com os filhos. Nos caucasianos e nos asiáticos este fenómeno não é característico, nem aceite na sua cultura.

\section{DURAÇÃO DO CASAMENTO}

Entre os diferentes grupos étnicos, nos E.U.A, a mais baixa idade para casar foi encontrada nos asiáticos. ${ }^{2}$

Os casamentos dos afro-americanos são os que du- ram menos tempo, seguidos dos dos hispânicos e dos brancos, sendo os dos asiáticos os de maior duração (dados do Center of Disease Control, 2000). Este efeito étnico é muito forte: dez anos após o casamento, um em cada dois casamentos negros, um em cada três casamentos brancos ou hispânicos e um em cada cinco casamentos entre asiáticos, nos EUA, dissolveram-se. ${ }^{2}$

\section{MÉTODOS CONTRACEPTIVOS E INFECÇÕES SEXUALMENTE TRANSMISSÍVEIS}

Segundo os dados de um estudo efectuado em 1996, em Glasgow, as asiáticas usam menos a pílula que as não asiáticas, independentemente do seu estado marital. Os homens asiáticos são os que usam menos o preservativo. A abstinência sexual nos asiáticos ocorre, frequentemente, por motivos religiosos, motivo que não é importante para os não asiáticos. O nível elevado de abstinência sexual entre os asiáticos, sobretudo na mulher, reflecte-se na baixa procura dos serviços de saúde, sendo utilizados, apenas, por aqueles que são sexualmente activos..$^{15}$

Os asiáticos têm menos infecções sexualmente transmitidas (blenorragia, sida) e uma das razões parece ser o início mais tardio da vida sexual, com menor número de parceiros sexuais ao longo da vida.

As asiáticas também recorrem menos aos serviços de saúde para efectuar rastreio do cancro do colo do útero ou da mama. Este grupo parece ter mais dificuldade em falar sobre a sua sexualidade, devendo-se a sua aparente modéstia a questões de ordem religiosa, em que, segundo as tradições teológicas, a actividade sexual está confinada ao casamento.

Os homens afro-americanos são os que têm um nível mais baixo de conhecimentos sobre contracepção, relativamente às mulheres da mesma origem e que apresentam as mais baixas taxas de utilização do preservativo, pelo que se espera que a transmissão das infecções sexualmente transmissíveis e da SIDA, entre heterossexuais, nas mulheres africanas, continue a aumentar. ${ }^{15}$ Como as mulheres africanas têm de lidar com as dificuldades em manter relações de longa duração e, simultaneamente, adoptar medidas de prevenção, muitas apercebem-se que têm muito pouco controlo sobre o comportamento sexual dos seus parceiros e oportunidades limitadas para introduzir a utilização do preservativo nas suas relações. ${ }^{14}$ Geralmente, deci- 
dem elas a escolha do método contraceptivo e preferem o implante progestativo ou o DIU, sendo muito problemático o uso da pílula. As mais jovens aderem melhor à pílula, no entanto, ainda com alguma irregularidade na sua toma.

A menarca surge mais tarde na mulher negra. Nalgumas comunidades festeja-se o momento em que a mulher já pode ter relações sexuais e ser mãe, daí o maior número de gravidezes nas idades adolescentes. No puerpério, não têm relações devido à crença de que os espermatozóides contaminam o leite materno. Actualmente, devido a esta crença e ao medo de perder a ligação afectiva, muitas preferem o aleitamento artificial ao materno.

Um estudo realizado no Reino Unido, sobre a relação entre origem étnica, vida sexual e infecções génito-urinárias, conclui que as mulheres negras são as que iniciam a vida sexual mais cedo, em média antes dos dezassete anos. Apresentam um número mais elevado de infecções, assim como um número mais elevado de gravidezes e de filhos.

As brancas referem maior número de parceiros sexuais, um maior número de parceiros não regulares e, consequentemente, uma sexualidade mais ocasional, usando, com maior probabilidade, o preservativo. ${ }^{15}$

Ainda segundo este estudo, as mulheres negras apresentam maior susceptibilidade às infecções por clamídia, gonococos e tricomonas. A percentagem elevada de homens negros com IST que não informa as suas parceiras é preocupante. Uns fazem-no por omissão, outros por desconhecimento da sua condição de infectados, muitas vezes por não recorrerem aos serviços ou consultas de saúde sexual e reprodutiva, que ainda são associadas, principalmente, às mulheres.

A sexualidade na comunidade cigana está associada à conjugalidade e a nupcialidade à procriação. Neste grupo social, a iniciação sexual e o início da procriação coincidem no tempo. Os homens não aceitam o uso de métodos contraceptivos, pelo que, se a mulher optar por um método, será sem o conhecimento do companheiro. Geralmente, optam pelo DIU ou por um progestageneo injectável. Sinais de mudança surgem nas mulheres com fraca ligação à tradição cigana, nas letradas e nas jovens, aproximando-se de alguns valores e comportamentos que caracterizam a sociedade portuguesa contemporânea, com descendência menos numero- sa, algum planeamento familiar das gravidezes, valorização do adiamento da idade de início da procriação e partilha conjugal da experiência da maternidade. ${ }^{16}$

\section{CIRCUNCISÃO FEMININA}

A circuncisão feminina, mutilação genital feminina ou «fanado», como é popularmente conhecida, é um procedimento frequente em comunidades de cultura islâmica e, em 1998, a OMS estimava entre 80 a 120 milhões o número de mulheres que tinham sido sujeitas a esta prática na infância ou puberdade. Nos Estados Unidos foi proibida desde 1996. ${ }^{2}$ Em Portugal não existem dados concretos, mas o nosso quadro legal pune a mutilação genital feminina. No entanto, é um país de risco para a execução desta prática, pois recebe imigrantes do continente africano, sobretudo oriundos da Guiné-Bissau, onde a prática da excisão feminina atinge os quarenta e cinco por cento. Os profissionais de saúde devem estar despertos para a existência deste fenómeno e precisam de formação para lidar com ele. Estão cientificamente documentadas as consequências da mutilação genital feminina na saúde mental, sexual e reprodutiva da mulher, com aumento da susceptibilidade ao VIH, além dos riscos obstétricos, com consequências fatais. ${ }^{17} \mathrm{Na}$ maioria dos casos, os efeitos imediatos resultam de infecções, abcessos e hemorragias locais. A infibulação (excisão do clítoris e dos pequenos lábios) pode ter efeitos mais duradouros e mais graves, incluindo infecções crónicas do tracto urinário e genital, obstrução à saída do fluxo menstrual e infertilidade. Durante o parto, o tecido cicatricial existente nas mulheres mutiladas pode romper e provocar hemorragias graves. As que foram sujeitas a infibulação e com os lábios vaginais fechados têm de ser excisadas para permitir o parto, após o que são novamente «fechadas», para assegurar o prazer dos maridos.

\section{CIRCUNCISÃO MASCULINA}

No mundo, um quarto dos homens são circuncidados. É uma prática antiga, aconselhada pelos Muçulmanos e Judeus. Pratica-se, também, por tradições não religiosas. Nos EUA, um terço dos homens é circuncidado. Na Europa, a taxa de circuncisões tem vindo a descer. Um estudo em Inglaterra, em 1990, revelou que 32 por cento dos homens dos 45 aos 59 anos tinha sido circuncidado, contra 12 por cento apenas no grupo dos 16 aos 
24 anos. $^{18}$

Segundo vários estudos, a circuncisão apresenta vantagens. Parece diminuir, em dez vezes, a frequência de infecções urinárias no rapaz (Herzog, 1989 eWiswell e col., 1987), permite corrigir a fimose, reduzir o risco de cancro do pénis, de IST e de SIDA (Moses e col., 1998) assim como diminuir a probabilidade de se infectar ou de transmitir a infecção por papilomavírus às parceiras, e destas contraírem cancro do colo do útero $(\mathrm{Ca}-$ tellsague e col., 2002). ${ }^{2,19}$

A desvantagem apontada, de que o homem ficaria com menor sensibilidade erótica do pénis, não se confirma, referindo Laumann, em 1997, ter encontrado uma associação positiva entre circuncisão e menor incidência de disfunção sexual no homem. ${ }^{2}$

\section{DUPLO PAPEL SEXUAL}

A igualdade de género e a saúde sexual e reprodutiva requerem a cooperação e participação dos homens. Em muitos países do mundo, são os homens que, usualmente, decidem o número e tipo de relacionamentos sexuais, a frequência da actividade sexual e a utilização de métodos contraceptivos, por vezes através de coacção ou de violência. É evidente que os homens devem estar activamente envolvidos, para se alcançar a igualdade de género e se conseguir uma saúde sexual e reprodutiva mais plena e baseada no respeito e reconhecimento dos direitos sexuais e reprodutivos. Para isso, é preciso ter em conta muitos factores culturais e sociais, pois a participação dos homens na saúde sexual e reprodutiva varia consoante os países e regiões de origem.

A saúde sexual e reprodutiva e a contracepção continuam a ser uma responsabilidade atribuída às mulheres. De facto, uma grande percentagem dos homens casados, em todo o mundo, refere nunca ter discutido planeamento familiar com as suas parceiras e menos de um terço é responsável pela contracepção. ${ }^{20}$

\section{CONCLUSÃO}

A escassez de estudos existentes em Portugal sobre os conhecimentos, crenças, atitudes e comportamentos na área da sexualidade das diferentes comunidades aqui residentes, dificulta aos técnicos de saúde um esclarecimento antecipado sobre estas questões. Este desconhecimento tem implicações na nossa prática clí- nica, pois dificulta "chegar» às diferentes comunidades e, assim, obter melhores indicadores de saúde sexual e reprodutiva.

\section{REFERÊNCIAS BIBLIOGRÁFICAS}

1. Lewis $L$ J. Examining sexual health discourses in a racial/ethnic context. Arch Sex Behav 2004 Jun; 33 (3): 223-34.

2. Levay S, Valente SM. Human sexuality. Sunderland, MA: Sinauer Associates; 2002.

3. Saavedra L, Magalhães S, Soares D, Ferreira S, Leitão F. Género, cultura e sexualidade em jovens portuguesas e portugueses: um programa de educação sexual. Comunicação apresentada no IV Congresso Astur-galaico de Sociologia; 2007 Mar; Corunha, Espanha.

4. Nodin N, coord. Os jovens portugueses e a sexualidade em finais do século XX. Lisboa: Associação para o Planeamento da Família; 2001.

5. Matos MG, Simões C, Tomé G, Silva M, Gaspar T, Diniz JA, et al. Aventura Social \& Saúde: indicadores de saúde dos adolescentes portugueses: Relatório Preliminar HBSC 2006. Disponível em: http://www. fmh.utl.pt/aventurasocial/pdf/Indicadores_de_Saude.pdf [acedido em 26/11/2008].

6. Dias SF, Matos MG, Gonçalves AC. Preventing HIV transmission in adolescents: an analysis of the Portuguese data from the Health Behaviour School-aged Children study and focus groups. Eur J Public Health 2005 Jun; 15 (3): 300-4.

7. Cuffee JJ, Hallfors DD, Waller MW. Racial and gender differences in adolescent sexual attitudes and longitudinal associations with coital debut. J Adolesc Health 2007 Jul; 41 (1): 19-26.

8. Hayes C. Risking the future: adolescent sexuality, pregnancy, and child bearing. Vol. I, Washington, DC: National Academy Press; 1987.

9. Barone C, Ickovics JR, Ayers TS, Katz SM, Voyce CK, Wieissberg RP. Highrisk sexual behaviour among young urban students. Fam Plan Perspect 1996 Mar-Apr; 28 (2): 69-74.

10. Gaspar T, Matos MG, Gonçalves A, Ferreira M, Linhares F. Comportamentos Sexuais, Conhecimentos e Atitudes face ao VIH/SIDA em Adolescentes Migrantes. Psicologia, Saúde e Doenças 2006;7 (2): 299-316.

11. Smith EA, Udry JR. Coital and non-coital sexual behaviours of white and black adolescents. Am J Public Health 1985 Oct; 75 (10): 1200-3.

12. Santos L, Goracci M, Gonçalves Y. Migrações Internacionais: género e saúde sexual e reprodutiva. Disponível em: www.apf.pt/cms/files/conteudos/file/migracoes.pdf [acedido em 26/11/08].

13. Bancroft J. Human sexuality and its problems. 2nd ed. Edimburgh: Churchill Livingstone; 1989.

14. Dias S, Matos MG, Gonçalves A. Comportamento sexual: auto-relatos numa comunidade migrante. Rev Port Pedagogia 2001; XXXV-2: 137-54 .

15. Bradby $\mathrm{H}$, Williams R. Behaviours and expectactions in relation to sexual intercourse among 18-20 year old Asian and non-Asians. Sex Transm Infect 1999 Jun; 75 (3): 162-7.

16. Santos A. Há muito remédio de agora não ter crianças: Representações e Práticas Contraceptivas de Mulheres Ciganas. Sexualidade \& Planeamento Familiar 2008 Jan-Jun; 48/49: 4-10.

17. ACIDI, I.P. Seis mil vítimas de mutilação genital por dia. Disponível em: http://www.acidi.gov.pt/modules.php? name $=$ New\&file $==$ article $\&$ si 
[acedido em 02-02-09].

18. Wellings K, Collumbien M, Slaymaker E, Singh S, Hodges Z, Patel D, et al. Sexual behaviour in context: a global perspective. The Lancet 2006 Nov 11; 368 (9548): 1706-28.

19. Ribeiro G. A circuncisão masculina está associada a um menor risco de infecção pelo VIH. Rev Port Clin Geral 2007 Jul-Ago; 23 (4): 482-4.

20. Marques RM, Frade A. Saúde sexual e reprodutiva e envolvimento masculino. Departamento de Cooperação e Desenvolvimento da APF. Disponível em:www.apf.pt/cms/files/conteudos/file/envol_masc.pdf [acedido em 26/11/09].

\section{ENDEREÇO PARA CORRESPONDÊNCIA}

Maria Fernanda Freire da Fonseca

Tlm: 936712502

E-mail: mfernandafonseca@sapo.pt

Marisa Cortez Lucas

Tlm: 919276210

E-mail:nb_ml@kanguru.pt

Recebido em 25/02/09

Aceite para publicação em 27/02/09

\section{ABSTRACT}

Sexual Behaviour is a complex reality. Our sexuality isn't only biologically determined as our entire social and cultural organization is also sexualized. In this paper, our goal is to approach some of the implications of ethnicity and culture on sexual behaviours, in order to evaluate their implications on the practice of health care providers. We believe that it is important to understand the complexity of sexual behaviour in the way it can influence our daily practice. In various periods of human life, it is possible to find certain elements that show us that culture and ethnicity have an important role on sexuality.

In adolescents, we can see that the feelings about sexual intercourse, use of condoms, number of sexual partners and unwanted pregnancy are associated with ethnicity and culture.

In adults, it is also possible to verify that ethnicity and culture influence sexual behaviours. When we approach, among others, themes like self body image, menstruation, marital sexuality, masturbation, STD, pregnancy, use of condoms, we see that ethnicity and culture are responsible for several differences in sexual behaviours.

On the other hand, we must understand that sexuality is ruled and influenced by society, with all the historical, demographic, cultural and economic changes that are associated with gender. Gender influences the behaviours of all ethnicities and cultures. For example, sexual and reproductive health and contraception are still the «responsibility» of women. All over the world, a large percentage of married men refer that they never have discussed this issue with their partners, and less than one third is responsible for contraception.

We can conclude that it is imperative that all health technicians became aware about the common beliefs and attitudes that affect sexual behaviours in different communities.

Keywords: Sexuality; Sexual Behaviour; Sexual Attitude; Sexual Health; Ethnicity. 